\title{
An Analysis of Polymorphisms Within the Wnt Signaling Pathway in Relation to Ovarian Cancer Risk in a Polish Population
}

\author{
Adrianna Mostowska $\cdot$ Piotr Pawlik $\cdot$ Stefan Sajdak $\cdot$ \\ Janina Markowska $\cdot$ Monika Pawałowska $\cdot$ \\ Margarita Lianeri $\cdot$ Paweł P. Jagodzinski
}

Published online: 28 September 2013

(C) The Author(s) 2013. This article is published with open access at Springerlink.com

\begin{abstract}
Background and Objective The Wnt/ $\beta$-catenin signaling pathway has been considered to be a factor in the development and progression of ovarian cancer.

Methods All patients with ovarian cancer and controls were tested for BRCAl mutations (5382incC, C61G, 4153delA) with HybProbe assays and for BRCA2 mutation (5946delT) using high-resolution melting curve analysis (HRM). Mutation carriers were excluded from the association analysis. We studied nine single nucleotide polymorphisms (SNPs) located in CTNNB1 ( $\beta$-catenin) [rs4533622, rs2953], APC (rs11954856, rs351771, rs459552), and AXIN2 (rs4074947, rs7224837, rs3923087, rs2240308) in women with ovarian cancer without BRCA1/ BRCA2 mutations $(n=228)$ and controls $(n=282)$. Genotyping of CTNNB1 rs4533622, rs2953, APC rs351771, AXIN2 rs4074947, rs3923087, and rs2240308 was performed by HRM, while that of APC rs11954856, rs459552 and AXIN2 rs7224837 was conducted by PCR
\end{abstract}

Electronic supplementary material The online version of this article (doi:10.1007/s40291-013-0059-y) contains supplementary material, which is available to authorized users.

A. Mostowska $\cdot$ M. Lianeri · P. P. Jagodzinski ( $\square)$ Department of Biochemistry and Molecular Biology, Poznań University of Medical Sciences, 6 Święcickiego St., 60-781 Poznan, Poland

e-mail: pjagodzi@am.poznan.pl

P. Pawlik - S. Sajdak

Clinic of Gynecological Surgery, Poznań University of Medical Sciences, Poznan, Poland

J. Markowska - M. Pawałowska Chair of Gynecologic Oncology, Poznań University of Medical Sciences, Poznan, Poland followed by the appropriate restriction enzyme digestion [PCR-restriction fragment length polymorphism (PCRRFLP)].

Results The most common BRCAl/BRCA2 mutations were identified in 30 patients with ovarian cancer. These mutations were not found in controls. The lowest $p$ values of the trend test $\left(p_{\text {trend }}\right)$ were observed for the APC rs351771 and rs11954856 SNPs in patients with ovarian cancer ( $p_{\text {trend }}=0.006$ and $p_{\text {trend }}=0.007$, respectively). Using a dominant inheritance model, we found that the $A P C$ rs11954856 SNP is associated with an increased risk of ovarian cancer development [odds ratio $=2.034(95 \%$ CI 1.302-3.178); $p=0.002]$. We also observed significant allelic differences for the APC rs351771 SNP between patients and controls $(p=0.006)$.

Conclusion Our study demonstrated significantly increased APC rs11954856 and rs351771 SNP frequencies in Polish women with ovarian cancer.

\section{Introduction}

Ovarian cancer has the highest mortality among gynecological malignancies in Europe and the USA [1,2]. Several factors, including genetic background, are known to increase the risk of ovarian cancer development [3]. To the well-recognized hereditary factors of ovarian cancer belong some of high-penetrance genes: BRCAl (3-6\%), BRCA2 (1-3\%), and HNPCC DNA mismatch repair genes (1-2 \%) [3-5]. However, these gene mutations explain less than $40 \%$ of the familial predisposition to ovarian cancer [6]. These data indicate that the missing heritability can be elucidated by a multigenic disease model with contribution of moderate and low-penetrance risk genes to ovarian malignancies [6]. 
Several intracellular pathways have been recognized in ovarian carcinogenesis [7]. Among them, the Wnt/ $\beta$-catenin/T-cell factor (TCF)/lymphoid enhancer factor (Lef) signaling pathway has been considered to be essential for the growth and progression of ovarian cancers [8]. The Wnt/ $\beta$-catenin signaling pathway regulates a variety of elementary cellular functions, such as proliferation, polarity, adhesion, and motility during development, differentiation, and adult tissue homeostasis [9]. This pathway is also essential for normal ovarian development, and different components of the canonical Wnt signaling pathway are presented in the ovary $[10,11]$. Wnt stimulation leads to a cytoplasmic accumulation of $\beta$-catenin that is subsequently translocated to the nucleus, where it interacts with the TCF family and induces the transcription of Wnt target genes [12]. The absence of Wnt stimulation results in $\beta$ catenin phosphorylation by components of the cytosol multi-protein degradation complex and its subsequent proteosomal degradation [12]. This multi-protein degradation complex contains adenomatous polyposis coli (APC), conductin (AXIN1 and AXIN2), glycogen synthase kinase-3 $\beta$ (GSK3 $\beta$ ), and casein kinase 1 (CK1) [12].

The components of the Wnt/ $\beta$-catenin/TCF/Lef signaling pathway have been reported to be up-regulated in various cancer types, including ovarian cancers [13, 14]. Moreover, many studies have demonstrated that the presence of mutations or polymorphisms in CTNNB1, APC, and $A X I N 2$ can lead to aberrant activation of $\mathrm{Wnt} / \beta$-catenin signaling at the onset of various types of malignancies, including ovarian cancer [15-31]. In addition to these findings, inactivation of the $A P C$ gene in mice leads to the deregulation of $\mathrm{Wnt} / \beta$-catenin signaling and the formation of adenocarcinomas that are morphologically similar to human ovarian endometrioid adenocarcinoma [32]. Therefore, we selected nine single nucleotide polymorphisms (SNPs) of CTNNB1, APC, and AXIN2 located in distinct blocks of linkage disequilibrium (LD) in order to study whether these SNPs can be genetic risk factors of ovarian cancer (Online Resource Supplemental Table 1 and Supplemental Fig. 1A, Fig. 1B and Fig. 1C.). Selected SNPs were tested in the group of ovarian cancer patients and controls who did not carry the most common mutations of the BRCA1/BRCA2 genes.

\section{Material and Methods}

\subsection{Patients and Controls}

The patients include 258 women with histologically determined ovarian carcinoma according to the International Federation of Gynecology and Obstetrics (FIGO). They were inducted into the study from the University
Hospital, Clinic of Gynecological Surgery and Chair of Gynecologic Oncology at Poznań University of Medical Sciences (Poznań, Poland). Histopathological classification, including the stage, grade, and tumor type, was performed by an experienced pathologist (Table 1). The control group included 282 unrelated healthy female volunteers who were matched by age to the female cancer patients. Controls were selected during medical examination at the University Hospital, Clinic of Gynecological Surgery at Poznań University of Medical Sciences (Table 1). Written informed consent was obtained from all participating individuals. The procedures of the study were approved by the Local Ethical Committee of Poznań University of Medical Sciences. All women with ovarian cancer and controls were Caucasian from the Wielkopolska area of Poland.

\subsection{Genotyping}

Genomic DNA was isolated from peripheral blood leukocytes by salt extraction.

All patients and controls with ovarian cancer were tested for the three most common BRCA1 mutations (5382incC, C61G, 4153delA) affecting the Polish population using the LightCycler ${ }^{\circledR} 480$ system with HybProbe assays (Roche, Indianapolis, IN, USA). In addition, the patient group was

Table 1 Clinical characteristics of ovarian cancer patients and healthy controls

\begin{tabular}{lll}
\hline Characteristic & Patients $(n=258)$ & Controls $(n=282)$ \\
\hline Mean age (years) \pm SD & $58.4 \pm 9.7$ & $57.4 \pm 7.5$ \\
Histological grade & \\
G1 & $83(32.2 \%)$ & \\
G2 & $85(32.9 \%)$ & \\
G3 & $90(34.9 \%)$ \\
Gx & $0(0.0 \%)$ \\
Clinical stage & \\
I & $96(37.2 \%)$ \\
II & $40(15.5 \%)$ \\
III & $88(34.1 \%)$ \\
IV & $34(13.2 \%)$ \\
Histological type & \\
Serous & $90(34.9 \%)$ \\
Mucinous & $30(11.6 \%)$ \\
Endometrioid & $48(18.6 \%)$ \\
Clear cell & $24(9.3 \%)$ \\
Brenne & $0(0.0 \%)$ \\
Mixed & $22(8.5 \%)$ \\
Solid & $18(7.0 \%)$ \\
Untyped carcinoma & $26(10.1 \%)$ \\
\hline
\end{tabular}

Data are given as no. (\%) unless otherwise stated 
tested for the presence of the most common BRCA2 mutation (5946delT) using high-resolution melting curve analysis (HRM) [Supplemental Table 2]. Information on HybProbe probe sequences is available upon request. The DNA samples were then genotyped for the nine SNPs in $C T N N B 1, A P C$, and AXIN2 (Supplemental Table 1 and Supplemental Fig. 1A, Fig. 1B, and Fig. 1C). SNPs were selected with the use of the genome browsers of the International HapMap Consortium (http://www.hapmap. org/index.html.en), University of California Santa Cruz (UCSC; http://genome.ucsc.edu), and dbSNP database (http://www.ncbi.nlm.nih.gov/projects/SNP/). SNPs were selected based on functional significance, location in different LD blocks, and minor allele frequency (MAF) $>0.1$ in the Caucasian population. Genotyping of the CTNNB1 rs4533622, rs2953, APC rs351771, and AXIN2 rs4074947, rs3923087, rs2240308 SNPs was performed by HRM on the LightCycler ${ }^{\circledR} 480$ system (Roche Diagnostics, Mannheim, Germany). Genotyping of the APC rs11954856, rs459552, and AXIN2 rs7224837 SNPs was performed by PCR, followed by the appropriate restriction enzyme digestion [PCR-restriction fragment length polymorphism (PCR-RFLP)] according to the manufacturer's instructions (Fermentas, Vilnius, Lithuania). Primer sequences and conditions for HRM and PCR-RFLP analyses are presented in Supplemental Table 2. Genotyping quality was evaluated by repeated genotyping of $10 \%$ randomly selected samples.

\subsection{Statistical Analysis}

For each SNP, the Hardy-Weinberg equilibrium (HWE) was assessed by Pearson's goodness-of-fit Chi-square $\left(\chi^{2}\right)$ statistic. The differences in the allele and genotype frequencies between cases and controls were determined using standard $\chi^{2}$ or Fisher tests. The odds ratio (OR) and associated $95 \%$ confidence intervals were also calculated. The data were analyzed under recessive and dominant inheritance models. For the additive inheritance model, SNPs were tested for association with ovarian cancer using the Cochran-Armitage trend test. To adjust for the multiple testing, the Single Nucleotide Polymorphism Spectral Decomposition (SNPpD) method was used [33]. Pair-wise LD between selected SNPs was computed as both $D^{\prime}$ and $r^{2}$ values using HaploView 4.0 software (Broad Institute, Cambridge, MA, USA). Haplotype analysis was performed using the UNPHASED 3.1.5 program with the following analysis options: all window sizes, full model, and uncertain haplotype [34]. Haplotypes with a frequency below 0.01 were set to zero. The $p$ values for both global and individual tests of haplotype distribution between cases and controls were determined. Statistical significance was assessed using the 1,000-fold permutation test.
High-order gene-gene interactions among all tested polymorphic loci were studied by the multifactor dimensionality reduction (MDR) approach (MDR version 2.0 beta 5). A detailed explanation on the MDR method has been described elsewhere [35]. Based on the obtained testing balanced accuracy and cross-validation consistency values, the best statistical gene-gene interaction models were established. A 1,000-fold permutation test was used to assess the statistical significance of MDR models (MDR permutation testing module 0.4 .9 alpha).

\section{Results}

\subsection{BRCA/BRCA2 Mutation Analysis}

In patients with ovarian cancer, 30 carriers of the most common BRCAl mutations were identified. The BRCAl 5382insC mutation was identified in 22 individuals, C61G in five individuals, and 4153delA in three individuals (Supplemental Table 3). None of the patients was a carrier of the BRCA2 5946delT mutation. Moreover, none of the controls had the tested BRCA1/BRCA2 nucleotide variants.

\subsection{Association of CTNNB1, APC, and AXIN2 Single Nucleotide Polymorphisms with Ovarian Cancer Development in Patients Without BRCA1/BRCA2 Mutations}

The distribution of $C T N N B 1, A P C$, and AXIN2 genotypes did not display deviation from HWE between patients and control groups $(p>0.05)$. The number of genotypes, ORs, and $95 \%$ confidence interval calculations for the nine $C T N N B 1, A P C$, and AXIN2 polymorphisms are shown in Table 2.

The lowest $p$ values of the trend test $\left(p_{\text {trend }}\right)$ were observed for the $A P C$ rs351771 and rs11954856 SNPs in patients with ovarian cancer ( $p_{\text {trend }}=0.006$ and $p_{\text {trend }}=0.007$, respectively) [Table 2]. Moreover, we observed that, in a dominant inheritance model, the APC rs11954856 SNP is associated with an increased risk of ovarian cancer development $[\mathrm{OR}=2.034$ (95 \% CI 1.302-3.178); $p=0.002]$. We also found significant allelic differences for the APC rs351771 SNP $(p=0.006)$ between patients and controls. The experiment-wide significance threshold required to keep Type I error rate at $5 \%$ was 0.006 (effective number of independent marker loci: 8.388). There was no association of the APC rs11954856 SNP (Supplemental Table 4) or other tested SNPs (not shown) with any histologic subtypes of ovarian cancer. Furthermore, none of the other eight $C T N N B 1, A P C$, and $A X I N 2$ polymorphisms displayed significant association with ovarian cancer development either in dominant, recessive, or additive inheritance models (Table 2). 
Table 2 Association of polymorphic variants of $C T N N B 1, A P C$, and AXIN2 with the risk of ovarian cancer

\begin{tabular}{|c|c|c|c|c|c|c|c|c|c|c|}
\hline Gene & rs no. & Alleles ${ }^{\mathrm{a}}$ & $\mathrm{MAF}^{\mathrm{b}}$ & $\begin{array}{l}\text { Genotypes } \\
\text { cases }^{\mathrm{c}}\end{array}$ & $\begin{array}{l}\text { Genotypes } \\
\text { controls }^{\mathrm{c}}\end{array}$ & $\begin{array}{l}p_{\text {genotypic }} \\
\text { value }\end{array}$ & $\begin{array}{l}p_{\text {trend }} \\
\text { value }\end{array}$ & $\begin{array}{l}p_{\text {allelic }} \\
\text { value }\end{array}$ & $\begin{array}{l}\mathrm{OR}_{\text {dominant }}(95 \% \\
\mathrm{CI})^{\mathrm{d}} ; p \text { value }\end{array}$ & $\begin{array}{l}\mathrm{OR}_{\text {recessive }}(95 \% \\
\mathrm{CI})^{\mathrm{e}} ; p \text { value }\end{array}$ \\
\hline CTNNB1 & rs4533622 & $\mathrm{a} / \mathrm{C}$ & 0.46 & $78 / 113 / 37$ & $90 / 122 / 70$ & 0.057 & 0.092 & 0.082 & $\begin{array}{l}0.901 \\
\quad(0.622-1.306) \\
0.583\end{array}$ & $\begin{array}{l}0.587 \\
\quad(0.376-0.915) \\
0.018\end{array}$ \\
\hline CTNNB1 & rs 2953 & $\mathrm{~g} / \mathrm{T}$ & 0.46 & $78 / 113 / 37$ & $90 / 122 / 70$ & 0.057 & 0.092 & 0.082 & $\begin{array}{l}0.901 \\
\quad(0.622-1.306) \\
0.583\end{array}$ & $\begin{array}{l}0.587 \\
\quad(0.376-0.915) \\
0.018\end{array}$ \\
\hline$A P C$ & rs11954856 & $\mathrm{g} / \mathrm{T}$ & 0.48 & $35 / 129 / 63$ & $76 / 141 / 64$ & 0.007 & 0.007 & 0.009 & $\begin{array}{l}2.034 \\
\quad(1.302-3.178) \\
0.002\end{array}$ & $\begin{array}{l}1.302 \\
(0.871-1.948) \\
0.198\end{array}$ \\
\hline$A P C$ & rs351771 & $\mathrm{c} / \mathrm{T}$ & 0.45 & $88 / 114 / 26$ & $86 / 139 / 57$ & 0.015 & 0.006 & 0.006 & $\begin{array}{l}0.698 \\
\quad(0.483-1.009) \\
0.055\end{array}$ & $\begin{array}{l}0.508 \\
\quad(0.308-0.839) \\
0.007\end{array}$ \\
\hline$A P C$ & rs 459552 & $\mathrm{a} / \mathrm{T}$ & 0.30 & $129 / 86 / 13$ & $142 / 108 / 32$ & 0.064 & 0.041 & 0.034 & $\begin{array}{l}0.778 \\
\quad(0.548-1.106) \\
0.161\end{array}$ & $\begin{array}{l}0.472 \\
\quad(0.242-0.923) \\
0.025\end{array}$ \\
\hline AXIN2 & rs4074947 & $\mathrm{C} / \mathrm{t}$ & 0.19 & $137 / 80 / 10$ & $182 / 89 / 10$ & 0.577 & 0.298 & 0.302 & $\begin{array}{l}1.208 \\
\quad(0.841-1.734) \\
0.306\end{array}$ & $\begin{array}{l}1.249 \\
(0.510-3.056) \\
0.626\end{array}$ \\
\hline AXIN2 & rs7224837 & $\mathrm{A} / \mathrm{g}$ & 0.15 & $161 / 61 / 6$ & $203 / 71 / 8$ & 0.917 & 0.801 & 0.799 & $\begin{array}{l}1.069 \\
(0.727-1.573) \\
0.733\end{array}$ & $\begin{array}{l}0.926 \\
\quad(0.316-2.708) \\
0.888\end{array}$ \\
\hline AXIN2 & rs3923087 & $\mathrm{a} / \mathrm{G}$ & 0.22 & $133 / 84 / 10$ & $171 / 97 / 14$ & 0.814 & 0.775 & 0.777 & $\begin{array}{l}1.089 \\
(0.763-1.555) \\
0.640\end{array}$ & $\begin{array}{l}0.882 \\
\quad(0.384-2.026) \\
0.767\end{array}$ \\
\hline AXIN2 & rs2240308 & $\mathrm{A} / \mathrm{g}$ & 0.49 & $67 / 115 / 46$ & $71 / 146 / 65$ & 0.510 & 0.254 & 0.260 & $\begin{array}{l}0.809 \\
\quad(0.546-1.197) \\
0.288\end{array}$ & $\begin{array}{l}0.844 \\
\quad(0.551-1.292) \\
0.434\end{array}$ \\
\hline
\end{tabular}

Statistically significant results are highlighted in bold

Experiment-wide significance threshold required to keep Type I error rate at $5 \%$ (Nyholt correction): 0.006 (effective number of independent marker loci: 8.388)

$M A F$ minor allele frequency, $O R$ odds ratio, $p_{\text {trend }} p$ values of the trend test

${ }^{a}$ Uppercase denotes the more frequent allele in the control samples

${ }^{b}$ Calculated from the control samples

c The order of genotypes: DD/Dd/dd ( $d$ is the minor allele in the control samples)

${ }^{\mathrm{d}}$ Dominant model: $\mathrm{dd}+\mathrm{Dd}$ vs. DD ( $\mathrm{d}$ is the minor allele)

e Recessive model: dd vs. Dd + DD ( $d$ is the minor allele)

\subsection{Association of CTNNB1, APC, and AXIN2} Haplotypes with Ovarian Cancer Development in Patients Without BRCA1/BRCA2 Mutations

Haplotype analysis of the studied CTNNB1, APC, and AXIN2 polymorphisms did not reveal SNP combinations associated with the risk of ovarian cancer development (Table 3). The lowest global $p=0.025$ was observed for haplotypes composed of the APC rs11954856 and rs351771 SNPs (Table 3). However, these results were not statistically significant when permutations were used to generate empiric $p$ values. The empiric $5 \%$ quintile of the best $p$ value after 1,000 permutations was 0.03904 for $C T N N B 1,0.00748$ for $A P C$, and 0.00888 for AXIN2 haplotypes. The CTNNB1 rs4533622 and rs2953 SNPs were in perfect LD (Supplemental Table 5). The SNPs situated in distinct regions of $A P C$ and $A X I N 2$ were either in strong or weak pairwise LD. This was calculated from the control samples, and had $D^{\prime}$ ranges of $0.401-0.988$ for APC SNPs and 0.002-1.000 for AXIN2 SNPs (Supplemental Table 5).

3.4 Multifactor Dimensionality Reduction Analysis of Gene-Gene Interactions Among the Studied $C T N N B 1, A P C$, and AXIN2 Polymorphisms

Exhaustive MDR analysis evaluating two- to four-loci combinations of all studied SNPs for each comparison did not demonstrate statistical significance in predicting susceptibility to ovarian cancer development (Table 4). The best combination of possibly interactive polymorphisms 
Table 3 Results of haplotype analysis of the CTNNB1, APC, and AXIN2 genes in patients with ovarian cancer

\begin{tabular}{lrl}
\hline Polymorphisms & $\chi^{2}$ & $\begin{array}{l}\text { Global } \\
p \text { value }\end{array}$ \\
\hline CTNNB1 $^{\text {a }}$ & 3.038 & 0.386 \\
rs4533622_rs2953 & & \\
APC & 9.352 & 0.025 \\
rs11954856_rs351771 & 6.945 & 0.074 \\
rs351771_rs459552 & 11.141 & 0.133 \\
rs11954856_rs351771_rs459552 & & \\
AXIN2 & 1.702 & 0.636 \\
rs4074947_rs7224837 & 0.245 & 0.970 \\
rs7224837_rs3923087 & 2.976 & 0.395 \\
rs3923087_rs2240308 & 1.739 & 0.973 \\
rs4074947_rs7224837_rs3923087 & 4.037 & 0.775 \\
rs7224837_rs3923087_rs2240308 & 7.383 & 0.946 \\
rs4074947_rs7224837_rs3923087_rs2240308 & & \\
\hline$\chi^{2}$ Chi-square & & \\
a Empirical 5 \% quantile of the best $p$ value: 0.03904 & \\
b Empirical 5 \% quantile of the best $p$ value: 0.00748 & \\
c Empirical 5 \% quantile of the best $p$ value: 0.00888 & \\
& &
\end{tabular}

was observed for rs4533622 of CTNNB1, rs11954856 of $A P C$, and rs7224837 and rs2240308 for AXIN2 (testing balanced accuracy $=0.5719$, cross validation consistency of 10 out of 10 , permutation test $p=0.068$ ).

\section{Discussion}

We found that the APC rs 11954856 polymorphism may be a risk factor of ovarian cancer in Polish population. To date, several polymorphisms in $A P C$ have been demonstrated to be risk factors of different cancers $[24,25,30$,

Table 4 Results of gene-gene interactions analyzed by multifactor dimensionality reduction method

\begin{tabular}{llll}
\hline Polymorphisms & $\begin{array}{l}\text { Testing } \\
\text { balanced } \\
\text { accuracy }\end{array}$ & $\begin{array}{l}\text { Cross } \\
\text { validation } \\
\text { consistency } \\
(\%)\end{array}$ & $p$ value \\
& & 60 & 0.356 \\
\hline CTNNB1_rs4533622, & 0.5418 & & \\
APC_rs11954856 & & 50 & 0.828 \\
CTNNB1_rs4533622, & 0.5041 & & \\
APC_rs351771, & & 100 & 0.068 \\
AXIN2_rs4074947 & & & \\
CTNNB1_rs4533622, & 0.5719 & & \\
APC_rs11954856, & & & \\
AXIN2_rs7224837, & & & \\
AXIN2_rs2240308 & &
\end{tabular}

\footnotetext{
${ }^{a}$ Significance of accuracy (empirical $p$ value based on 1,000 permutations)
}

31]. The rs454886 SNP (Supplemental Fig. 1B) has been associated with increased breast cancer risk [24]. The APC I1307K rs1801155 polymorphism (Supplemental Fig. 1B) has been observed to be associated with an increased risk for colorectal cancer but not breast/ovarian cancers [25, 36, 37]. Moreover, a significant interaction has been found between the D1822V rs459552 (Supplemental Fig. 1B) polymorphism and dietary intakes of high fat, cholesterol, calcium, and fiber for colorectal cancer risk [30, 31].

The $\beta$-catenin is the main Wnt pathway effector for which aberrant action has been demonstrated in various cancers [13]. The role of mutations in CTNNB1 leading to aberrant functioning of $\beta$-catenin at the onset of ovarian cancer have been highlighted. There are several studies demonstrating the presence of an oncogenic mutation, mainly located in exon 3 of $C T N N B 1$, in upwards of $40 \%$ ovarian endometrioid adenocarcinomas [15-17, 38, 39]. Additionally, several polymorphisms in CTNNB1 (rs4135385, rs11564475, rs2293303) have been correlated with gastric cancer susceptibility and favorable gastric cancer survival (rs4135385) (Supplemental Fig. 1A) [19]. However, in our studies we did not observe a significant association of the selected CTNNB1 SNPs with the development of ovarian cancer.

AXIN is a scaffold protein in the destruction complex, enabling the phosphorylation of $\beta$-catenin by GSK-3 $\beta[40$, 41]. Recently, a $64 \%$ allele-specific loss of heterozygosity in AXIN2 was observed in epithelial ovarian cancer [42]. However, in our studies we did not find an association of the studied AXIN2 SNPs with ovarian cancer development. Certain polymorphisms located in AXIN2 have been shown to be risk factors of astrocytoma (rs1133683), lung cancer (rs2240308), and prostate cancer (rs35285779) [Supplemental Fig. 1C] [26-29]. In addition to these findings, the five AXIN2 SNPs rs7210356, rs4791171, rs11079571, rs3923087, and rs3923086 were associated with an increased risk of breast cancer (Supplemental Fig. 1C) [24].

\section{Conclusion}

Our study demonstrated significantly increased $A P C$ rs11954856 and rs351771 SNPs frequencies in Polish women with ovarian cancer. These SNPs were not identified in recently conducted genome-wide association studies (GWAS) as risk factors for ovarian cancer, probably due to genome-wide significance thresholds required for a conventional GWAS ( $p$ value $<5 \times 10^{-8}$ ) [43-45]. The association of intronic rs11954856 and synonymous rs351771 (Ala545Ala) SNPs with ovarian cancer may be due to $\mathrm{LD}$ with one or more functional polymorphisms of $A P C$. The lack of association of CTNNB1 and AXIN2 SNPs 
with ovarian cancer might be due to the small sample size. Therefore, to confirm the role of the studied SNPs in ovarian cancer, this study should be replicated in a larger and independent cohort, and functional studies of these SNPs must be performed.

Acknowledgments Supported by grant no. 502-01-0112418207474, Poznań University of Medical Sciences.

Conflict of Interest The authors declare that they have no conflict of interest.

Open Access This article is distributed under the terms of the Creative Commons Attribution Noncommercial License which permits any noncommercial use, distribution, and reproduction in any medium, provided the original author(s) and the source are credited.

\section{References}

1. Ferlay J, Autier P, Boniol M, et al. Estimates of the cancer incidence and mortality in Europe in 2006. Ann Oncol. 2007;18(3):581-92.

2. Jemal A, Siegel R, Ward E, et al. Cancer statistics, 2008. CA Cancer J Clin. 2008;58(2):71-96.

3. Sueblinvong T, Carney ME. Current understanding of risk factors for ovarian cancer. Curr Treat Options Oncol. 2009;10(1-2): 67-81.

4. Berchuck A, Schildkraut JM, Marks JR, et al. Managing hereditary ovarian cancer risk. Cancer. 1999;86(11 Suppl):2517-24.

5. Frank TS, Manley SA, Olopade OI, et al. Sequence analysis of BRCA1 and BRCA2: correlation of mutations with family history and ovarian cancer risk. J Clin Oncol. 1998;16(7):2417-25.

6. Holschneider $\mathrm{CH}$, Berek JS. Ovarian cancer: epidemiology, biology, and prognostic factors. Semin Surg Oncol. 2000;19(1): 3-10.

7. Choi YP, Shim HS, Gao MQ, et al. Molecular portraits of intratumoral heterogeneity in human ovarian cancer. Cancer Lett. 2011;307(1):62-71.

8. Bitler BG, Nicodemus JP, Li H, et al. Wnt5a suppresses epithelial ovarian cancer by promoting cellular senescence R. Cancer Res. 2011;71(19):6184-94.

9. Chien AJ, Conrad WH, Moon RT. A Wnt survival guide: from flies to human disease. J Invest Dermatol. 2009;129(7):1614-27.

10. Hsieh M, Johnson MA, Greenberg NM, et al. Regulated expression of Wnts and Frizzleds at specific stages of follicular development in the rodent ovary. Endocrinology. 2002;143(3): 898-908.

11. Vainio S, Heikkila M, Kispert A, et al. Female development in mammals is regulated by Wnt-4 signalling. Nature. 1999; 397(6718):405-9.

12. Novak A, Dedhar S. Signaling through beta-catenin and Lef/Tcf. Cell Mol Life Sci. 1999;56(5-6):523-37.

13. Herr P, Hausmann G, Basler K. WNT secretion and signalling in human disease. Trends Mol Med. 2012;18(8):483-93.

14. Gatcliffe TA, Monk BJ, Planutis $\mathrm{K}$, et al. Wnt signaling in ovarian tumorigenesis. Int J Gynecol Cancer. 2008;18(5):954-62.

15. Sagae S, Kobayashi K, Nishioka Y, et al. Mutational analysis of beta-catenin gene in Japanese ovarian carcinomas: frequent mutations in endometrioid carcinomas. Jpn J Cancer Res. 1999;90(5):510-5.

16. Saegusa M, Okayasu I. Frequent nuclear beta-catenin accumulation and associated mutations in endometrioid-type endometrial and ovarian carcinomas with squamous differentiation. J Pathol. 2001;194(1):59-67.

17. Wu R, Zhai Y, Fearon ER, et al. Diverse mechanisms of betacatenin deregulation in ovarian endometrioid adenocarcinomas. Cancer Res. 2001;61(22):8247-55.

18. Polakis P. Wnt signaling in cancer. Cold Spring Harb Perspect Biol. 2012;4:a008052.

19. Wang S, Tian Y, Wu D, et al. Genetic variation of CTNNB1 gene is associated with susceptibility and prognosis of gastric cancer in a Chinese population. Mutagenesis. 2012;27(6):623-30.

20. Starker LF, Fonseca A, Akerström G, et al. Evidence of a stabilizing mutation of $\beta$-catenin encoded by CTNNB1 exon 3 in a large series of sporadic parathyroid adenomas. Endocrine. 2012;42(3):612-5.

21. Yoshie T, Nishiumi S, Izumi Y, et al. Regulation of the metabolite profile by an APC gene mutation in colorectal cancer. Cancer Sci. 2012;103(6):1010-21.

22. Erbilgin $\mathrm{Y}, \mathrm{Ng} \mathrm{OH}$, Mavi $\mathrm{N}$, et al. Genetic alterations in members of the Wnt pathway in acute leukemia. Leuk Lymphoma. 2012;53(3):508-10.

23. Sercan Z, Pehlivan M, Gokturk D, et al. Beta-catenin mutations are not observed in chronic myeloid leukemia. Tumori. 2009;95(6):836-9.

24. Wang X, Goode EL, Fredericksen ZS, et al. Association of genetic variation in genes implicated in the beta-catenin destruction complex with risk of breast cancer. Cancer Epidemiol Biomarkers Prev. 2008;17(8):2101-8.

25. Laken SJ, Peterson GM, Gruber SB, et al. Familial colorectal cancer in Ashkenazim due to a hypermutable tract in APC. Nat Genet. 1997;17(1):79-83.

26. Pinarbasi E, Gunes EG, Pinarbasi $\mathrm{H}$, et al. AXIN2 polymorphism and its association with prostate cancer in a Turkish population. Med Oncol. 2011;28(4):1373-8.

27. Gunes EG, Pinarbasi E, Pinarbasi H, et al. Strong association between lung cancer and the AXIN2 polymorphism. Mol Med Report. 2009;2(6):1029-35.

28. Gunes EG, Pinarbasi E, Pinarbasi H. AXIN2 polymorphism and its association with astrocytoma in a Turkish population. Mol Med Report. 2010;3(4):705-9.

29. Kanzaki H, Ouchida M, Hanafusa H, et al. Single nucleotide polymorphism of the AXIN2 gene is preferentially associated with human lung cancer risk in a Japanese population. Int J Mol Med. 2006;18(2):279-84

30. Wong HL, Peters U, Hayes RB, et al. Polymorphisms in the adenomatous polyposis coli (APC) gene and advanced colorectal adenoma risk. Eur J Cancer. 2010;46(13):2457-66.

31. Guerreiro CS, Cravo ML, Brito M, et al. The D1822V APC polymorphism interacts with fat, calcium, and fiber intakes in modulating the risk of colorectal cancer in Portuguese persons. Am J Clin Nutr. 2007;85(6):1592-7.

32. Wu R, Hendrix-Lucas N, Kuick R, et al. Mouse model of human ovarian endometrioid adenocarcinoma based on somatic defects in the Wnt/beta-catenin and PI3K/Pten signaling pathways. Cancer Cell. 2007;11(4):321-33.

33. Nyholt DR. A simple correction for multiple testing for SNPs in linkage disequilibrium with each other. Am J Hum Genet. 2004;74(4):765-9.

34. Dudbridge F. Likelihood-based association analysis for nuclear families and unrelated subjects with missing genotype data. Hum Hered. 2008;66(2):87-98.

35. Hahn LW, Ritchie MD, Moore JH. Multifactor dimensionality reduction software for detecting gene-gene and gene-environment interactions. Bioinformatics. 2003;19(3):376-82.

36. Abrahamson J, Moslehi R, Vesprini D, et al. No association of the I1307K APC allele with ovarian cancer risk in Ashkenazi Jews. Cancer Res. 1998;58(14):2919-22. 
37. Gershoni-Baruch R, Patael Y, Dagan, et al. Association of the I1307K APC mutation with hereditary and sporadic breast/ovarian cancer: more questions than answers. $\mathrm{Br} \mathrm{J}$ Cancer. 2000;83(2):153-5.

38. Wright K, Wilson P, Morland S, et al. Beta-catenin mutation and expression analysis in ovarian cancer: exon 3 mutations and nuclear translocation in $16 \%$ of endometrioid tumours. Int $\mathrm{J}$ Cancer. 1999;82(5):625-9.

39. Sarrió D, Moreno-Bueno G, Sánchez-Estévez C, et al. High frequency of beta-catenin mutations in borderline endometrioid tumours of the ovary. Expression of cadherins and catenins correlates with distinct histologic types of ovarian carcinomas. Hum Pathol. 2006;37(5):1042-9.

40. Eklof Spink K, Fridman SG, Weis WI. Molecular mechanisms of beta-catenin recognition by adenomatous polyposis coli revealed by the structure of an APC-beta-catenin complex. EMBO J. 2001;20(22):6203-12.
41. Nakamura T, Hamada F, Ishidate T, et al. Axin, an inhibitor of the Wnt signalling pathway, interacts with beta-catenin, GSK-3 beta and APC and reduces the beta-catenin level. Genes Cells. 1998;3(6):395-403.

42. Notaridou M, Quaye L, Dafou D, et al. Common alleles in candidate susceptibility genes associated with risk and development of epithelial ovarian cancer. Int J Cancer. 2011;128(9):2063-74.

43. Song H, Ramus SJ, Tyrer J, et al. A genome-wide association study identifies a new ovarian cancer susceptibility locus on 9p22.2. Nat Genet. 2009;41(9):996-1000.

44. Goode EL, Chenevix-Trench G, Song H, et al. A genome-wide association study identifies susceptibility loci for ovarian cancer at $2 \mathrm{q} 31$ and $8 \mathrm{q} 24$. Nat Genet. 2010;42(10):874-9.

45. Bolton KL, Tyrer J, Song H, et al. Common variants at 19p13 are associated with susceptibility to ovarian cancer. Nat Genet. 2010;42(10):880-4. 